\title{
Aproximación a la diversidad liquénica del Parque Natural del Cadí-Moixeró. Líquenes de los bosques de coníferas
}

\author{
Esteve Llop ${ }^{1}$ y Pere Aymerich ${ }^{2}$
}

\begin{abstract}
Resumen: Llop, E. \& Aymerich, P. 2014. Aproximación a la diversidad liquénica del Parque Natural del Cadí-Moixeró. Líquenes de los bosques de coníferas. Bot. Complut. 38: 29-34.

El estudio de la diversidad de los líquenes epífitos, lignícolas y terrícolas de tres localidades del Parc Natural del Cadí-Moixeró, representativas de tres tipos diferentes de bosques (Pinus uncinata, Abies alba y mixto de P. sylvestris y $P$. uncinata), ha permitido elaborar un catálogo de 65 taxones, 29 de los cuales son novedad para el Parque, y de estos 8 son nueva cita para Cataluña (Cladonia asahinae, C. novochlorophaea, Cliostomum tenerum, Lecanora confusa, Lepraria rigidula, Micarea bauschiana, Peltigera neopolydactyla y Trapeliopsis gelatinosa). Se compara la diversidad liquénica entre los tres bosques en base a la composición de la biota y la riqueza específica. El grado de madurez forestal y el grado de continentalidad inciden en las diferencias que se observan entre las tres localidades.
\end{abstract}

Palabras clave: biodiversidad, hongos liquenizados, epífitos, lignícolas, terrícolas, Península Ibérica, Pirineos orientales.

Abstract: Llop, E. \& Aymerich, P. 2014. Contribution to the lichen diversity of the Natural Park Cadí-Moixeró. Lichens of conifer forests. Bot. Complut. 38: 29-34.

The diversity on epiphytic, lignicolous and terricolous lichens from three localities from the Natural Park Cadí-Moixeró, representing three different sorts of forest (Pinus uncinata, Abies alba and a mixture of P. sylvestris and P. uncinata), has yielded a catalogue of 65 taxa. Among them, 29 are new for the protected area, and 8 of those are new quotations for Catalonia (Cladonia asahinae, C. novochlorophaea, Cliostomum tenerum, Lecanora confusa, Lepraria rigidula, Micarea bauschiana, Peltigera neopolydactyla, and Trapeliopsis gelatinosa). The lichen diversity of the three sorts of forest has been compared in terms of biota composition and species richness. Forest maturity and the conditions of continentality play some role on the differences observed between the localities.

Key words: biodiversity, lichenized fungi, epiphytic, lignicolous, terricolous, Iberian Peninsula, eastern Pyrenees.

\section{INTRODUCCIÓN}

El Parque Natural del Cadí-Moixeró es una de las zonas protegidas catalanas que ocupan una mayor extensión, unos $433 \mathrm{~km}^{2}$ aproximadamente. Este conjunto de sierras calcáreas incluye una buena representación del paisaje propio del Prepirineo oriental. La variación altitudinal del parque, desde los $800 \mathrm{~m}$ hasta los $2.648 \mathrm{~m}$ en su punto culminante, permite una gran diversidad de comunidades, estando representadas desde las típicas del piso mediterráneo submontano hasta las del piso alpino. La peculiar ubicación del sistema montañoso, orientado de oeste a este, determina una diferenciación climática importante entre la vertiente norte y sur, así como una marcada continentalidad en el sector más occidental.

La biota liquénica del Parque Natural del Cadí-Moixeró es uno de los elementos más desconocidos de la biodiver- sidad de este espacio protegido. La primera cita de un liquen de la sierra del Cadí se remonta a finales del siglo XIX, cuando Brodoa intestiniformis fue citada por Vayreda (1882) como Parmelia encausta. Hasta el momento no se dispone de un catálogo de los líquenes del Parque, y solo hay constancia de datos parciales, generalmente simples citas, incluidas en artículos centrados en esta área (Gómez-Bolea \& Hladun 1981, Hladun 1982, Alvaro \& Hladun 1983), en trabajos con un alcance territorial más amplio (Gómez-Bolea 1985, Martínez 2002) 0 en revisiones de algunos grupos taxonómicos (Hladun 1984, Martínez \& Burgaz 1988, Martínez \& Hafellner 1998, Giralt 2001, Burgaz 2009, Knudsen et al. 2009, Navarro-Rosinés et al. 2009). El listado actual de líquenes del parque incluye a 74 taxones (Hladun 2013).

Dada la gran diversidad de ecosistemas presentes en el Parque, se ha propuesto el estudio de la biota liquénica

\footnotetext{
${ }^{1}$ Dpt. Biologia Vegetal-Botànica, Universitat de Barcelona, Avda. Diagonal 645, 08028 Barcelona, España. ellv66@gmail.com

${ }^{2}$ Barcelona 29, 08600 Berga, España.

Recibido: 30 agosto 2013. Aceptado: 13 septiembre 2013.
} 
de los bosques de coníferas, por ser las comunidades que ocupan mayor superficie y por ser uno de los elementos más representativos dentro del parque. Además, se ha seleccionado este tipo de hábitats porque la conservación de los bosques maduros es una de las líneas de gestión dentro de este espacio protegido.

\section{MATERIALES Y MÉTODOS}

La selección de las localidades ha tenido en cuenta dos tipos de criterios. Por una parte que se tratara de masas con cierto grado de madurez forestal. Se ha considerado como indicadores de madurez la presencia de árboles con un diámetro considerable, una estructura heterogénea de clases de edad y una buena disponibilidad de madera muerta (en general, en forma de tocones de árboles cortados varias décadas atrás). El segundo criterio a considerar fue que incluyeran una representación de las tres especies de coníferas que forman bosques en el parque (pino albar, pino negro y abeto).

Se seleccionaron tres localidades:

1. Els Orris (Bagà, Barcelona), UTM (ED50) 409062 4683690; 1.900-1.980 m; orientación W-NW; bosque de pino negro (Pinus uncinata) maduro relativamente denso, con pocos arbustos en el sotobosque.

2. Muntanya de Riu (Bellver de Cerdanya, Lleida), UTM (ED50) 4010814685264 ; 1.700-1.750 m; orientación NW; bosque de abetos (Abies alba) cerrado y denso, con pocos arbustos en el sotobosque.

3. Obaga de Adraén (la Vansa i Fórnols, Lleida), UTM (ED50) 378641 4681648; 1.760-1.810 m; orientación W-NW; bosque mixto de pino albar (Pinus sylvestris) y pino negro muy abierto, con abundancia de Juniperus communis y Buxus sempervirens en el sotobosque.

La superficie muestreada en cada localidad corresponde a 1-2 ha. Se ha estudiado la diversidad liquénica de troncos, ramas, tocones y suelos. El material recolectado fue examinado e identificado según las metodologías habituales basadas en las observaciones macroscópicas y microscópicas de los caracteres. La correcta determinación de algunos taxones ha requerido la aplicación de técnicas de cromatografía en capa fina para identificar las substancias liquénicas (Elix \& Ernst-Russell 1993, Orange et al. 2001). El material recolectado se ha depositado en el herbario BC. La nomenclatura de los taxones sigue a Nimis \& Martellos (2008) y Smith et al. (2009).

Se ha comparado la composición de la biota liquénica de las tres localidades en base a la morfología y ecología. Además, la diversidad entre los tres tipos de bosques se ha comparado en base al coeficiente de similaridad de Sørensen.

\section{RESULTADOS}

El listado de los líquenes identificados en las tres 10calidades comprende 65 taxones. Para cada taxón se in- dica la localidad, el substrato sobre el cual se ha identificado, las substancias liquénicas si han sido identificadas, y entre paréntesis el número o números de herbario correspondientes. Las primeras citas para Cataluña se indican en *.

Buellia griseovirens (Turner et Borrer ex Sm.) Almb. -3: ramas de Buxus sempervirens (930041). Citada previamente por Gómez-Bolea \& Hladun (1981) y Gómez-Bolea (1985).

Caloplaca alnetorum Giralt, Nimis \& Poelt -3: ramas de Rhamnus alpinus, ramas de Juniperus communis (930046, 930047, 930048, 930049, 930050, 930052).

Caloplaca cerina (Hedw.) Th. Fr. -3: ramas de Rhamnus alpinus (930046, 930047). Citada previamente por Gómez-Bolea (1985).

Caloplaca ferruginea (Huds.) Th. Fr. -3: ramas de Juniperus communis, ramas de Rhamnus alpinus, ramas de Buxus sempervirens, ramas de pino (930038, 930043, 930046, 930047, 930049, 930052); -2: tronco de Salix caprea (30143). Citada previamente por Gómez-Bolea \& Hladun (1981) y Gómez-Bolea (1985).

Caloplaca hungarica H. Magn. -2: ramas de Abies alba (930159, 930162, 930181); -1: ramas y ramitas de Pinus uncinata (930134, 930135, 930137). Citada previamente por Gómez-Bolea \& Hladun (1981) y Gómez-Bolea (1985).

Caloplaca lucifuga G. Thor -3: ramas de Juniperus communis (930050).

Candelariella vitellina (Ehrh.) A. Massal. -3: ramas de Rhamnus alpinus, ramas de Buxus sempervirens (930038, 930046, 930047); -2: ramas de Abies alba, tronco y ramas de Salix caprea (930143, 930144, 930162). Citada previamente por Gómez-Bolea \& Hladun (1981) y Gómez-Bolea (1985).

Candelariella xanthostigma (Pers. ex Ach.) Lettau -3: ramas de Juniperus communis, ramas de Rhamnus alpinus, ramas de Buxus sempervirens $(930038,930041,930042,930046,930048,930049$, 930050, 930051,930052). Citada previamente por Gómez-Bolea (1985).

Catillaria nigroclavata (Nyl.) J. Steiner -3: rama gruesa de Juniperus communis (930052).

Cetraria islandica (L.) Ach. -1: en suelo y base de tronco de Pinus uncinata (930126). Citada previamente por Gómez-Bolea \& Hladun (1981) y Álvaro \& Hladun (1983).

*Cladonia asahinae J. W. Thomson -1: tocón de Pinus uncinata (930130). Presenta los ácidos fumarprotocetrárico, rangifórmico (mayoritarios), norrangifórmico, protocetrárico y confumarprotocetrárico (minoritarios).

Cladonia chlorophaea (Flörke ex Sommerf.) Spreng. -3: tocón de pino (930037); -2: tocón de Abies alba (930160, 930166, 930167, 930172, 930175); -1: tocón de Pinus uncinata (930072, 930129, 930131, 930132). Presenta los ácidos fumarprotocetrárico (mayoritario), protocetrárico y confumarprotocetrárico (minoritarios). Citada previamente por Álvaro \& Hladun (1983).

Cladonia coniocraea (Flörke) Spreng. -3: tocón de pino (930036, 930037); -2: tocón de Abies alba, terrícola (930154, 930160, 930166);-1: tocón de Pinus uncinata (930127). Presenta los ácidos fumarprotocetrárico (mayoritario), protocetrárico y confumarprotocetrárico (minoritarios). Citada previamente por GómezBolea \& Hladun (1981), Álvaro \& Hladun (1983) y Gómez-Bolea (1985).

Cladonia digitata (L.) Hoffm. -3: tocón de pino (930035, 930037); -2: tocón de Abies alba, terrícola (930145, 930171);-1: tocón de 
Pinus uncinata $(930121,930129)$. Presenta el ácido tammnólico. Citada previamente por Álvaro \& Hladun (1983).

Cladonia fimbriata (L.) Fr. -2: tocón de Abies alba, terrícola (930157, 930168, 930169, 930170, 930175); -1: tocón de Pinus uncinata, terrícola $(930071,930139)$. Presenta los ácidos fumarprotocetrárico (mayoritario), protocetrárico y confumarprotocetrárico (minoritarios). Citada previamente por Álvaro \& Hladun (1983).

Cladonia furcata (Huds.) Schrad. -2: terrícola (930173). Presenta los ácidos fumarprotocetrárico (mayoritario), protocetrárico y confumarprotocetrárico (minoritarios). Citada previamente por Álvaro \& Hladun (1983).

*Cladonia novochlorophaea (Sipman) Brodo \& Ahti -1: tocón y base de tronco de Pinus uncinata (930121, 930136, 930140). Presenta los ácidos homosekikaico y sekikaico (mayoritarios) y fumarprotocetrárico, protocetrárico y confumarprotocetrárico (minoritarios).

Cladonia pyxidata (L.) Hoffm. -2: terrícola (930150, 930158, 930171). Presenta los ácidos fumarprotocetrárico (mayoritario), protocetrárico y confumarprotocetrárico (minoritarios). Citada previamente por Gómez-Bolea \& Hladun (1981) y Álvaro \& Hladun (1983).

*Cliostomum tenerum (Nyl.) Coppins \& S. Ekman- 3: tocón de pino (930034).

Fuscidea lightfootii (Sm.) Coppins \& P. James -1: base de tronco de Pinus uncinata $(930062,930121)$.

Hypogymnia farinacea Zopf -2: base del tronco, ramas y ramitas de Abies alba, tronco de Salix caprea (930142, 930152, 930153, 930159, 930181); -1: base de tronco, ramas y ramitas de Pinus uncinata $(930123,930133,930134,930135,930137)$.

Hypogymnia physodes (L.) Nyl. -2: base del tronco, tronco y ramas de Abies alba (930148, 930151, 930156, 930165, 930178); -1: base de tronco de Pinus uncinata $(930066,930070)$. Citada previamente por Gómez-Bolea \& Hladun (1981) y Gómez-Bolea (1985).

Hypogymnia tubulosa (Schaer.) Hav. -2: ramas de Abies alba (930162); -1: base del tronco de Pinus uncinata (930053, 930058, 930062, 930067). Citada previamente por Gómez-Bolea \& Hladun (1981).

Imshaugia aleurites (Ach.) S. L. F. Mey. -3: tronco de pino (930039, 930044). Citada previamente por Gómez-Bolea (1985) como Parmeliopsis aleurites.

Lecania naegelii (Hepp) Diederich \& van den Boom -3: ramas de $J u$ niperus communis $(930049,930050)$. Citada previamente por Gómez-Bolea (1985).

Lecanora carpinea (L.) Vain. -3: ramas de Buxus sempervirens, tocón de pino (930033, 930042); -2: ramas de Abies alba, tronco y ramas de Salix caprea (930143, 930144, 930159, 930181). Citada previamente por Gómez-Bolea \& Hladun (1981) y Gómez-Bolea (1985).

Lecanora chlarotera Nyl. -3: ramas de Buxus sempervirens, ramas de Juniperus communis (930041, 930050, 930051, 930052). Citada previamente por Álvaro \& Hladun (1983) y Gómez-Bolea (1985).

*Lecanora confusa Almb. -2: ramas de Abies alba (930159, 930162, 930181); -1: tocón, ramas y ramitas de Pinus uncinata (930128, 930133, 930134, 930135, 930137, 930141).

Lecanora hagenii (Ach.) Ach. -3: ramas de Rhamnus alpinus, ramas de Juniperus communis, ramas de Buxus sempervirens (930038, 930046, 930047, 930048, 930049, 930050, 930052).

Lecanora horiza (Ach.) Röhl. -3: ramas de Juniperus communis $(930049,930050,930052)$.

Lecanora leptyrodes (Nyl.) Degel. -3: ramas de Rhamnus alpinus, ramas de Juniperus communis, ramas de Buxus sempervirens
(930038, 930046, 930047, 930050). Citada previamente por Gómez-Bolea \& Hladun (1981) y Gómez-Bolea (1985).

Lecanora mughicola Nyl. -1: rama de Juniperus communis muerto (930065).

Lecanora pulicaris (Pers.) Ach. -3: ramas de Rhamnus alpinus, ramas de Buxus sempervirens, ramas de Juniperus communis (930038, 930041, 930042, 930046, 930047, 930048, 930049, 930050, 930051,); -2: tronco y ramas de Abies alba, tronco y ramas de Salix caprea (930143, 30144, 930155, 930159, 930162, 930181); -1: tronco, ramas y ramitas de Pinus uncinata $(930053,930058$, 930059, 930062, 930067, 930124, 930125, 930133, 930134, 930135, 930137). Citada previamente por Gómez-Bolea (1985).

Lecanora varia (Hoffm.) Ach. -1: base de tronco de Pinus uncinata (930070, 930121).

Lecidella elaeochroma (Ach.) M. Choisy-3: ramas de Rhamnus alpinus, ramas de Buxus sempervirens, ramas de Juniperus communis, ramas de pino (930038, 930041, 930042, 930043, 930046, 930047, 930048, 930049, 930050, 930051, 930052); -2: ramas de Abies alba, ramas de Salix caprea (930144, 930159, 930181). Citada previamente por Gómez-Bolea \& Hladun (1981) y Álvaro \& Hladun (1983) como L. euphorea, y Gómez-Bolea (1985).

Lepraria eburnea J. R. Laundon -3: ramas de Juniperus communis (930040); -2: tocón de pino (930149). Presenta los ácidos alectoriálico y protocetrárico.

*Lepraria rigidula (B. de Lesd.) Tønsberg -1: rama muerta de Juniperus communis (930065). Presenta atranorina y el ácido nefrosteránico.

Melanelixia fuliginosa subsp. glabratula (Lamy) J. R. Laundon; -2: tronco y ramas de Abies alba $(930147,930155)$.

Melanohalea exasperatula (Nyl.) 0. Blanco, A. Crespo, Divakar, Essl., D. Hawksw. \& Lumbsch -3: ramas de Buxus sempervirens, ramas de Juniperus communis, ramas de pino (930038, 930041, 930043 , 930049, 930050, 930051, 930052); -2: ramitas de Abies alba (930153); -1: ramas y ramitas de Pinus uncinata (930133, 930134, 930135, 930137). Citada previamente por Gómez-Bolea \& Hladun (1981), Álvaro \& Hladun (1983) y Gómez-Bolea (1985) como Parmelia exasperatula.

*Micarea bauschiana (Körb.) V. Wirth et Vězda -1: tocón de Pinus uncinata (930128).

Micarea misella (Nyl.) Hedl. -2: tocón de Abies alba (930168).

Micarea peliocarpa (Anzi) Coppins -1: base de tronco y ramas de $P i$ nus uncinata $(930058,930059,930062,930067,930121,930138)$.

Parmeliopsis ambigua (Wulfen) Nyl. -3: tocón y tronco de pino (930033, 930034, 930039, 930044); -2: base del tronco de Abies alba (930146, 930151, 930152); -1: tocón, base del tronco y ramas de Pinus uncinata $(930053,930056,930058,930059,930062$, 930121, 930127, 930136, 930138, 930140). Citada previamente por Gómez-Bolea \& Hladun (1981), Álvaro \& Hladun (1983) y Gómez-Bolea (1985).

Parmeliopsis hyperopta (Ach.) Vain. -3: tocón de pino (930033); -1: tocón, base del tronco y ramas de Pinus uncinata $(930057,930058$, 930059, 930063, 930127, 930138). Citada previamente por Álvaro \& Hladun (1983).

Peltigera degenii Gyeln. -2: terrícola y en suelo acumulado sobre roca silícea $(930163,930177)$. No contiene substancias liquénicas.

*Peltigera neopolydactyla (Gyeln.) Gyeln. -2: en suelo acumulado sobre roca silícea (930176). Presenta tenuiorina, metilgiroforato, ácido girofórico, peltidactilina, dolicorrizina y zeorina.

Peltigera rufescens (Weiss) Humb. -2: terrícola (930179). Citada previamente por Martínez \& Hafellner (1998). 
Pertusaria hemisphaerica (Flörke) Erichsen -3: rama gruesa de Juniperus communis (930052).

Physcia adscendens (Fr.) H. Olivier -3: ramas de Rhamnus alpinus, ramas de Buxus sempervirens, ramas de pino (930038, 930043, 930046, 930047); -2: ramitas de Abies alba, tronco de Salix caprea $(930143,930153)$. Citada previamente por Gómez-Bolea \& Hladun (1981) y Gómez-Bolea (1985).

Pseudevernia furfuracea (L.) Zopf-3: ramas de Buxus sempervirens, ramas de Juniperus communis, tronco de pino (930038, 930041, 930045, 930048, 930052); -2: tronco, ramas y ramitas de Abies alba $(930153,930159,930161,930162,930180,930181)$; -1: base del tronco, tronco, ramas y ramitas de Pinus uncinata ( 930053 , 930058, 930062, 930064, 930067, 930070, 930121, 930122, 930124, 930125, 930133, 930134, 930135 930137). Citada previamente por Gómez-Bolea \& Hladun (1981) y Gómez-Bolea (1985).

Ramalina farinacea (L.) Ach. -2: tronco y ramas de Abies alba (930174). Citada previamente por Gómez-Bolea \& Hladun (1981) y Gómez-Bolea (1985).

Ramalina fastigiata (Pers.) Ach. -2: tronco y ramas de Abies alba (930164). Citada previamente por Gómez-Bolea (1985).

Ramalina fraxinea (L.) Ach. -1: ramas de Pinus uncinata (930124).

Ramalina pollinaria (Westr.) Ach. -2: tronco y ramas de Abies alba (930164). Presenta atranorina y los ácidos evérnico, obtusático y úsnico. Citada previamente por Gómez-Bolea (1985).

Rinodina orculata Poelt \& M. Steiner; Adraén: ramas de Rhamnus alpinus $(930046,930047)$. Citada previamente por Giralt (2001).

Rinodina septentrionalis Malme -3: ramas de pino (930043); -2: ramas de Salix caprea (930144). Citada previamente por Giralt (2001).

Scoliciosporum sarothamni (Vain.) Vězda -3: rama gruesa de Juniperus communis, ramas de Buxus sempervirens (930038, 930052); -2: ramas de Abies alba $(930159,930181)$.

Scoliciosporum umbrinum (Ach.) Arnold -2: tronco y ramas de Salix caprea (930143, 930144); -1: ramitas de Pinus uncinata (930134, 930137). Citada previamente por Gómez-Bolea (1985).

Tephromela atra (Huds.) Hafellner -3: ramas de Buxus sempervirens, ramas de Juniperus communis $(930038,930048)$. Citada previamente por Gómez-Bolea \& Hladun (1981) y Gómez-Bolea (1985) como Lecanora atra.

*Trapeliopsis gelatinosa (Flörke) Coppins \& P. James -1: tocón y base del tronco de Pinus uncinata (930059, 930063, 930121, 930140).

Usnea glabrescens (Nyl. ex Vain.) Vain. -1: tronco y ramas de Pinus uncinata (930122). Presenta los ácidos psorómico, 2'-oxo-dimetilpsorómico y úsnico.

Usnea hirta (L.) Weber ex F. H. Wigg. -2: tronco y ramas de Abies alba $(930149,930180) ;-1$ : tronco y ramas de Pinus uncinata $(930054,930055,930060,930061,930069,930125)$. Se han observado tres razas químicas, una raza solo presenta ácido úsnico, la segunda raza presenta el complejo del ácido murólico y ácido úsnico, y la tercera raza presenta los complejos del ácido estíctico y murólico y ácido úsnico. Citada previamente por Gómez-Bolea (1985).

Vulpicida pinastri (Scop.) J.-E. Mattsson \& M. J. Lai-1: base del tronco de Pinus uncinata (930121, 930126). Citada previamente por Gómez-Bolea \& Hladun (1981) y Gómez-Bolea (1985) como Cetraria pinastri.

Xanthoria parietina (L.) Th. Fr. -3: ramas de Rhamnus alpinus, ramas de Buxus sempervirens, ramas de Juniperus communis (930038,
930041, 930046, 930047, 930050). Citada previamente por Gómez-Bolea \& Hladun (1981) y Gómez-Bolea (1985).

Xylographa parallela (Ach.) Fr. -1: tocón y madera de Pinus uncinata $(930068,930141)$. Citada previamente por Álvaro \& Hladun (1983) como X. abietina.

\section{DISCUSIÓN}

Del total de especies identificadas, 29 son nuevas citas para el Parque. De estas, 8 taxones corresponden a nuevas aportaciones a la biota liquénica de Cataluña: Cladonia asahinae, C. novochlorophaea, Cliostomum tenerum, Lecanora confusa, Lepraria rigidula, Micarea bauschiana, Peltigera neopolydactyla y Trapeliopsis gelatinosa. Estos taxones representan el $12 \%$ del total de taxones identificados. La localidad con un mayor número de nuevas citas para Cataluña es Els Orris, con 6, Muntanya de Riu tiene dos y Adraén solo una.

La riqueza específica de las diferentes localidades es bastante parecida, 30 taxones en Els Orris, y 33 taxones tanto en Muntanya de Riu como en Adraén. La composición de la biota liquénica está dominada por los taxones con talo crustáceo, aunque hay diferencias significativas según la localidad. Las localidades de Els Orris, con bosque de pino negro, y Muntanya de Riu, con bosque de abetos, presentan una biota liquénica integrada por una proporción de taxones con talo crustáceo, foliáceo y fruticuloso bastante equilibrada. La localidad de Adraén, situada en un bosque mixto de pino albar y pino negro, más abierto que en las otras dos localidades, presenta un predominio de líquenes con talo crustáceo, con un porcentaje muy bajo de líquenes con talo fruticuloso (Fig. 1).

También varia la preferencia por el substrato entre las tres localidades. Destaca la ausencia de líquenes terrícolas en Adraén, presentando también una menor proporción de líquenes lignícolas. Por otra parte, destaca el elevado porcentaje de líquenes terrícolas en Muntanya de Riu, y la importancia de los líquenes lignícolas en Els Orris (Fig. 2). Si se compara el porcentaje de especies colonizadoras presentes en los tres bosques, Adraén cuenta con un 66,7\%, Muntanya de Riu el 30,3\% y Els Orris solo el $10 \%$. Las variaciones en estos valores estaría relacionado con el grado de madurez de las masas forestales. Los bosques más maduros presenta un porcentaje de especies colonizadoras más bajo, y por otra parte el porcentaje de taxones lignícolas se incrementa, debido a una mayor disponibilidad de este tipo de substrato. La presencia de más especies colonizadoras en Adraén estaría relacionado con su ubicación en el extremo occidental del parque, 


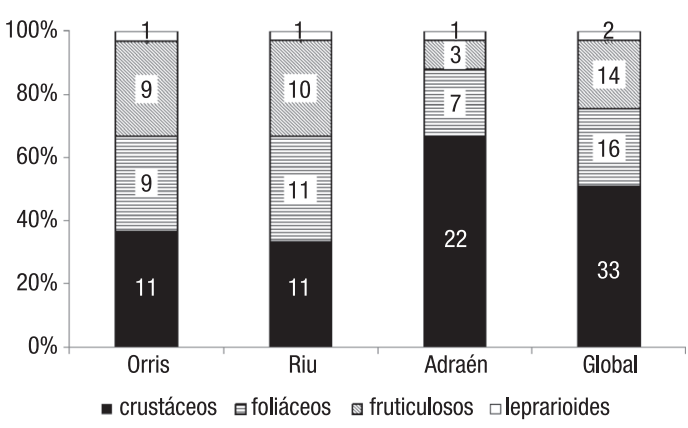

Fig. 1-Distribución de la morfología de los talos de la biota liquénica de las localidades estudiadas, así como del global del catálogo. Los valores indican el valor absoluto para cada tipo de talo presente.

bajo unas condiciones de mayor continentalidad, que determinaría unas perturbaciones climáticas más acusadas, y también por ser el bosque más abierto que favorece unas condiciones de mayor radiación solar y menor humedad.

La comparación entre la composición específica de las tres localidades mediante el coeficiente de similitud de Sørensen, no muestra un patrón tan claro como la composición de la biota liquénica. Las localidades de Els Orris y de Muntanya de Riu son las que presentan un valor del coeficiente más alto $(0,476)$. Por otra parte el coeficiente entre Els Orris y Adraén es bajo $(0,254)$, parecido a las diferencias observadas en la presencia de líquenes colonizadores y lignícolas. Pero el coeficiente entre Muntanya de Riu y Adraén no indica que haya tanta diferencia $(0,455)$, cuando sería de esperar unos valores más parecidos a los que aparecen entre Els Orris y Adraén. Quizás la ubicación de Muntanya de Riu en La Cerdanya, con unas características climáticas más continentales que Els Orris determina esta mayor similitud de la biota liquénica con Adraén.

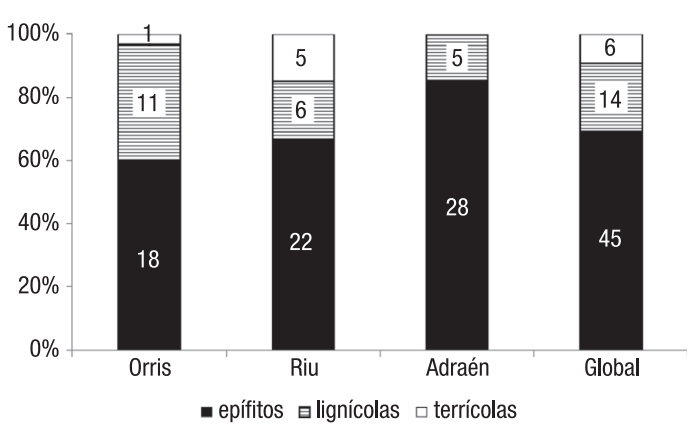

Fig. 2- Distribución de la ecología de las especies observadas en las localidades estudiadas, así como del global del catá$\log _{0}$. Los valores indican el valor absoluto para cada ecología.

\section{CONCLUSIONES}

El estudio de la diversidad liquénica en los bosques de coníferas del Parque Natural del Cadí-Moixeró ha permitido incrementar el conocimiento de la biota liquénica en 29 taxones, y además ha añadido 8 nuevos taxones al catálogo de líquenes de Cataluña.

La diversidad liquénica de los bosques maduros se traduce en la presencia de especies interesantes, como son los líquenes lignícolas, y también con la presencia de una mayor número de nuevas citas. El conocimiento de la biota es un elemento interesante a tener en cuenta por los responsables de los espacios protegidos para diseñar las prioridades en la gestión.

\section{AGRADECIMIENTOS}

Este trabajo ha sido auspiciado y financiado por la dirección del Parc Natural del Cadí-Moixeró, con el objectivo de mejorar el conocimiento de la diversidad liquénica y mejorar su conservación y gestión. Agradecemos a M. E. López de Silanes y A. R. Burgaz la revisión del manuscrito.

\section{BIBLIOGRAFÍA}

Álvaro, I.; Hladun, N. L. 1983. Observaciones sobre la colonización briológico-liquénica de la madera en descomposición en los bosques del Moixeró (Catalunya). Collect. Bot. (Barcelona) 14: 19-25.

Burgaz, A. R. 2009. El género Cladonia en la península Ibérica. Supergrupo Cocciferae. Bot. Complut. 33: 9-28.

Elix, J.A. \& ERNST-RusselL, K. D. 1993. A catalogue of standardized thin layer chromatographic data and biosynthetic relationships for lichen substances. 2nd Edn. Australian National University, Canberra.

GiRaLT, M. 2001. The lichen genera Rinodina and Rinodinella (lichenized Ascomycetes, Physciaceae) in the Iberian Peninsula. Bibl. Lichenol. 79: 1-160.
Gómez-Bolea, A. 1985. Líquenes epífitos en Cataluña. Centre Publ. Univ. Barcelona, Barcelona.

Gómez-Bolea, A.; Hladun, N. L. 1981. Datos para la flora liquénica de la Sierra del Cadí. I. Folia Bot. Misc. 2: 25-33.

HLadun, N. L. 1982. Omphalina umbellifera (L. ex Fr.) Quél. novedad para la flora liquénica española. Collect. Bot. (Barcelona) 13: 323-324.

HLadun, N. L. 1984. Contribución al conocimiento de los Coniocarpales (Líquenes) de Cataluña. Anales Biol., Fac. Biol., Univ. Murcia 1: 245-247.

HLadun, N. L. 2013. Mòdul Liquencat. Banc de Dades de Biodiversitat de Catalunya. Generalitat de Catalunya i Universitat de Barcelona. http://biodiver.bio.ub.es/biocat/homepage.html 
Knudsen, K.; Breuss, O. \& KocourKova, J. 2009. A new species of Placocarpus (Verrucariaceae) from Southern California. Lichenologist 41(6): 627-630.

MARTíNEZ, I. 2002. Lichenicolous fungi from the Iberian Peninsula and the Macaronesian area. Nova Hedwigia 74: 51-67.

Martínez, I. \& Burgaz, A. R. 1988. Revision of the genus Solorina (Lichenes) in Europe based on spore size variation. Ann. Bot. Fenn. 35: 137-142.

MarTínez, I.; Hafellner, J. 1998. Lichens and lichenicolous fungi on Peltigerales in the Iberian Peninsula and the $\mathrm{Ca}$ nary Islands. Mycotaxon 69: 271-310.

Navarro-Rosinés, P.; Calatayud, V. \& Hafellner, J. 2009. Contributions to a revision of the genus Cercidospora (Dothideales) 1. Species on Megasporaceae. Mycotaxon 110: 5-25.
Nimis, P. L. \& Martellos, S. 2008. ITALIC -The Information System on Italian Lichens. Version 4.0. University of Trieste, Dept. of Biology, IN4.0/1. http://dbiodbs.univ.trieste.it/ Orange, A.; James, P. W. \& White, F. J. 2001. Microchemical methods for the identification of lichens. British Lichen Society, London.

Smith, C. W.; Aptroot, A.; Coppins, B. J.; Flechter, A.; GilBert, O. L.; James, P. W. \& Wolseley, P. A. 2009. The Lichen Flora of Great Britain and Ireland. Natural History Museum Publications \& British Lichen Society, London.

VAYREDA, E. 1882. Catàlech de la Flora de la Vall de Núria. Associació Excursionista de Catalunya, Barcelona. 